\title{
Escolaridade materna: correlação com os indicadores obstétricos
}

\author{
Maternal educational level: \\ correlation with obstetric indicators
}

Fátima Hussein Haidar 1

Urânia Fernandes Oli vei ra 1

Luiz Fernando Costa Nascimento 1

\footnotetext{
1 Departamento de Medicina, Universidade de Taubaté Av. Tiradentes 500 Taubaté, SP 12080-130
}

\begin{abstract}
Theaim of this study was to estimate associations between level of maternal education as the dependent variable and several variables present in birth certificates under the official Brazilian Ministry of Health model. A total of 3,843 birth certificates were analyzed from Guaratinguetá, São Paul o State, for singleton hospital deliveries in 1998. Statistically significant associations were found between low maternal educational level and low birth weight, 3 or more live bi rths, history of still bi rth, and prenatal care including up to 6 visits. No association was found between abortions and preterm delivery as described in other studies, perhaps due to insufficient information. Maternal educational level can thus be considered an obstetric marker for some risk factors for the mother and infant.

Key words Women's Health; Risk Factors; Educational Status

Resumo O obj eti vo deste estudo é estimar as associações entre a escolari dade materna, como variável dependente, eal gu mas variávei s constantes na Declaração de Nascido Vivo (DNV) do Ministério da Saúde. Em uma análise de 3.843 DNV da regi ão de Guaratinguetá, São Paulo, relativas a partos hospitalares e nascimentos únicos, ocorridos no ano de 1998, foram encontradas associações estati sticamente si gnificativas entre a menor escolari dade e ocorrência de baixo peso ao nascer; número de fil hos vivos igual ou maior que três; história pregressa de fil hos mortos; maior número de partos e consultas médicas no pré-natal em número até seis. Não se observaram associ ações entre escol ari dade e aborto e tempo de gestação, citadas em outros trabal hos. Assim, a escol ari dade materna pode ser considerada um marcador obstétri co de risco para a gestante eo recém-nasci do.

Palavras-chave Saúdeda Mulher; Fatores de Risco; Escolaridade
\end{abstract}


Introdução

A escolaridade materna tem sido apresentada, como variável independente, em trabalhos epidemiológicos que abrangem os mais variados temas. Assim, a baixa escolaridade materna está associada a um risco maior de mortalidade materna (Orach, 2000; ThemeFilha et al., 1999). Também a morte fetal está associada à baixa escolaridade materna, como mostrado em trabal ho com mais de oitocentos mil nascimentos, o qual utilizou-se de um banco de dados do Centro Latino-Americano de Perinatologia, entre 1985 e 1997 (Conde-Agudelo et al., 2000).

Foi mostrada uma associação entre a maternidade precoce e baixo peso ao nascer e escolaridade menor das mães em uma amostra de 9.141 recém-nascidos americanos (Okosun et al., 2000), assi $m$ como em estudo realizado na Grécia (Lekea-Karanika et al., 1999). A prematuridade foi estudada por Astolfi \& Zonta (1999) que, examinando uma amostra de nascidos vivos na Itália entre 1990-1994, encontraram, através de análise univariada, uma alta associação de recém-nascidos prematuros, entre outras variáveis, com a baixa escolaridade materna.

Quanto à mortalidade infantil, há um aumento desta com a baixa escolaridade materna (Almeida et al., 1999; Bohland \& Jorge, 1999). O intervalo intergenésico, - isto é, entre os partos -, considerado como fator importante para a saúde de mães e filhos, está aumentado entre aquelas com maior escolaridade, mostrado em um trabalho na zona rural da Arábia Saudita com 332 mães (Al-Nahedh, 1999). Da mesma forma, o aleitamento materno e, principalmente, a oferta do colostro têm uma menor prevalência entre as mães com menor grau de instrução (Naj dawi \& Faouri, 1999; Okolo et al., 1999). Osborn et al. (2000) não encontraram associação entre aborto espontâneo e grau de instrução materna em uma anál ise na Itália, entre 1974 e 1995.

Quanto ao tipo de parto, a escolaridade maior está associada, neste grupo de mulheres, a uma maior taxa de parto cesáreo, sugerindo a existência de outros fatores além dos clínicos (Gould et al., 1989; Nascimento, 1997).

Os objeti vos deste trabalho são estudar as possíveis associações entre a escolaridade materna e as variáveis presentes na Declaração de Nascidos Vivos (DNV) do Ministério da Saúde (MS), na região de Guaratinguetá, São Paulo.
Material e métodos

Trata-se de um estudo do tipo transversal, realizado na região de Guaratinguetá, a qual se situa no médio Vale do Paraíba Paulista, com uma população estimada em 210.000 habitantes. Foram analisadas as DNV da Direção Regional de Saúde (DIR) XXIV, relativas ao ano de 1998, que estavam arquivadas no subgrupo de Guaratinguetá.

Os critérios de inclusão no estudo foram que os campos referentes à instrução materna estivessem preenchidos e não contivessem a informação "Ignorado". Estas DNV eram referentes a partos hospitalares e nascimentos únicos.

A variável dependente foi escolaridade materna, categorizada em ensino fundamental incompleto (menos de oito anos de escolaridade, incluindo-se as analfabetas) - categoria de referência -; ensino fundamental completo (oito anos de escolaridade); ensino médio (9 a 11 anos de escolaridade) e nível universitário (12 anos ou mais). $O$ peso ao nascer foi categorizado em baixo peso - peso menor que $2.500 \mathrm{~g}$ - e peso normal; o tipo de parto, em parto normal e parto cesáreo; número de consultas apresentou-se dividido em até seis consultas e mais que seis consultas no pré-natal; classificou-se o aborto prévio em ausente ou presente; filhos vivos (incluindo esta gravidez), em um ou dois e três e mais; filhos mortos, em ausente ou presente, não importando o número; número de partos, em primípara ou multípara, que resultou da soma das variáveis fil hos vivos e filhos mortos; tempo de gestação, considerado de termo para aqueles nascidos com 37 ou mais semanas de gestação e pré-termos.

A análise estatística utilizada foi a do teste de associação do $\chi^{2}$ entre as escolaridades e as demais variáveis; $\chi^{2}$ de tendência linear para estimar o comportamento das variáveis em relação à escolaridade materna, ordenada da menor para a maior, e odds ratio (OR) para estimar as razões de chances de ocorrência dos eventos, segundo os efeitos das escolaridades maiores quando comparadas com a categoria de referência, isto é, mães com menor escolaridade. Foram construídos intervalos de confiança (IC) de $95 \%$ e a significância estatística foi de $5 \%$

Os dados foram compilados e analisados pelo programa Epi Info versão 6.01 (Dean et al., 1994).

A tabela constante na seção Resultados refere-se aos dados dos partos ocorridos no ano de 1998, obtidos junto ao subgrupo de Guaratinguetá da DIR XXIV. 


\section{Resultados}

Foram incluídas neste estudo 3.843 DNV que continham os campos referentes ao grau de instrução da mãe preenchidos e a informação não era "Ignorado", uma possi bilidade contemplada na DNV. A idade materna média foi 24,6 anos com desvio padrão de 6,2 anos; a idade mínima foi 13 anos e a máxima, 50. Pode ser observado, na Tabela 1, que alguns totais são diferentes de 3.843, em virtude de al gumas variáveis terem sido excluídas por não apresentarem o preenchimento completo.

O teste de associação do $\chi^{2}$ com três graus de liberdade mostrou associação estatisticamente significativa entre a escolaridade materna, em categorias, e todas as variáveis, exceto para o tempo de gestação.

A Tabela 1 mostra a distribuição dos valores numéricos das variáveis e as respectivas percentagens, segundo a escolaridade materna, com os respectivos $p$, segundo $o \chi^{2}$ de tendência linear, OR e IC $95 \%$.

\section{Discussão}

A DNV do MS é um documento oficial que pode ser fonte importante de informações tanto da mãe, como do recém-nascido.

Pode ser observado que a menor escolaridade materna se apresenta associada à ocorrência de recém-nascido de baixo peso, como citado por Okosun et al. (2000). As mães com menos de oito anos de escolaridade têm uma chance 1,5 vez maior de terem recém-nascidos com baixo peso. Esta associação pode estar relacionada ao baixo padrão sócio-econômico destas mães, que possivel mente apresentam menor ganho de peso na gestação, início mais tardio do pré-natal ou qualidade deste, pois o número de consultas não foi fator de confusão ou interação para esta variável.

A escolaridade materna está fortemente associada ao tipo de parto quando as mães com maior grau de instrução apresentam uma chance seis vezes maior de terem seus filhos de parto cesáreo. Isso parece ser decorrente tanto de opção da mãe, como também médica, pois, como o parto cesáreo costuma ter um custo financeiro maior, as mães com maior escolaridade, que costumam ter melhores condições econômicas, podem optar por ele.

O número de consultas no pré-natal também se mostrou associado à escolaridade materna; as mães com maior instrução tinham uma chance duas vezes maior de efetuarem mais de seis consultas no pré-natal, sugerindo que este teria iniciado mais precocemente; assim, estas mães dariam maior importância ao pré-natal e/ ou teriam um acesso mais fácil ao acompanhamento de sua gestação. A DNV não fornece dados tanto do início do pré-natal, como do local onde ele foi realizado, se em serviço público ou privado.

As mães com menor escolaridade têm mais que três filhos quando comparadas com mães com maior escolaridade; esse fato pode estar associado a um menor intervalo intergenésico o que pode predispor estas crianças a riscos. $\mathrm{O}$ número de filhos maior que três pode ser decorrente de falta de informação ou falta deacesso aos serviços de saúde; as mães com maior escolaridade têm uma chance três vezes maior de terem até dois filhos, quando comparadas com aquelas com ensino fundamental incompleto. A queda nas taxas de natal idade, então, ocorrem no segmento da população mais privilegiado, que pode adotar medidas anticoncepcionais mais eficazes.

A associação das mortalidades perinatal, neonatal e infantil às mães com menor escolaridade (Bohland \& Jorge, 1999; Conde-Agudelo et al., 2000) pode decorrer não só do menor conhecimento destas mães quanto à importância do pré-natal, do intervalo intergenésico maior, do acompanhamento de rotina de seu recémnascido, como também do acesso mais difícil aos serviços de puericultura, em virtude de uma menor condição social por causa da baixa escolaridade.

$\mathrm{Na}$ DNV, o campo referente a filhos mortos não especifica a época de ocorrência do evento, se perinatal, neonatal precoce ou tardia ou pós-neonatal ; é permitido supor que essa variável dependa mais diretamente das mortalidades perinatal, neonatal precoce e tardia, uma vez que os dados de 1998 relativos à nossa região mostram que estas mortalidades contribuem com $75 \%$ dos óbitos que ocorrem abaixo de um ano de idade (SES-SP, 1998). Mesmo assim, é uma informação que poderia ser mais bem explicitada na DNV.

O maior número de partos, que foi obtido pela soma das variáveis filhos vivos e filhos mortos, também está associado à baixa escolaridade, com chance de ocorrer no mínimo três vezes maior, quando comparado com o número de partos das mães com mais de oito anos de escolaridade, o que corresponde aos níveis médio e superior. Como visto anteriormente, a falta de informação por uma possível dificuldade de acesso aos serviços de pré-natal, fato este associado ou não à fal ta de interesse da própria mulher, pode causar um menor conhecimento de métodos anticoncepcionais; Esta 
Tabela 1

Distribuição dos valo res numéricos das variáveis, com as porcentagens, de acordo com a escolaridade materna, dividida em quatro categorias, das OR, IC $95 \%$ e $p$, segundo o $\chi^{2}$ de tendência linear.

\begin{tabular}{|c|c|c|c|c|c|}
\hline & PGI (\%) & PGC (\%) & SG $(\%)$ & TG $(\%)$ & Total \\
\hline Baixo peso* & $220(10,7)$ & $62(7,8)$ & $57(7,3)$ & $13(6,7)$ & \\
\hline Peso normal & $1.844(89,3)$ & $733(92,2)$ & $727(92,7)$ & $181(93,3)$ & $3.837^{* *}$ \\
\hline OR (IC 95\%) & & $0,71(0,52-0,96)$ & $0,66(0,48-0,90)$ & $0,60(0,32-1,09)$ & \\
\hline \multicolumn{6}{|l|}{$p<0,01$} \\
\hline Parto normal* & $946(45,9)$ & $287(36,4)$ & $206(26,4)$ & $24(12,4)$ & \\
\hline Parto cesáreo & $1.116(54,1)$ & $501(63,6)$ & $575(73,6)$ & $170(87,6)$ & $3.825^{* *}$ \\
\hline OR (IC 95\%) & & $1,48(1,25-1,76)$ & $2,37(1,97-2,85)$ & $6,00(3,81-9,52)$ & \\
\hline \multicolumn{6}{|l|}{$p=0,000 \ldots$} \\
\hline Número de consultas $\leq 6 *$ & $746((36,6)$ & $247(31,7)$ & $214(29,8)$ & $42(24,0)$ & \\
\hline $\mathrm{N}$ úmero de consultas > 6 & $1.294(63,4)$ & $532(68,3)$ & $505(70,2)$ & $133(76,0)$ & $3.732^{* *}$ \\
\hline OR (IC 95\%) & & $0,81(0,67-0,91)$ & $0,74(0,61-0,89)$ & $0,55(0,38-0,79)$ & \\
\hline \multicolumn{6}{|l|}{$p<0,01$} \\
\hline Filhos vivos 1-2 & $1.299(62,8)$ & $657(82,6)$ & $679(86,4)$ & $164(84,5)$ & \\
\hline Filhos vivos 3 e mais* & $768(37,2)$ & $138(17,4)$ & $107(13,6)$ & $30(15,5)$ & $3.842 * *$ \\
\hline OR (IC 95\%) & & $2,81(2,28-3,47)$ & $3,75(2,98-4,72)$ & $3,23(2,13-4,92)$ & \\
\hline \multicolumn{6}{|l|}{$p=0,000 \ldots$} \\
\hline Filhos mortos ausentes & $1.948(94,2)$ & $772(90,9)$ & $762(96,9)$ & $190(97,9)$ & \\
\hline Filhos mortos presentes* & $119(5,8)$ & $22(9,1)$ & $24(3,1)$ & $4(2,1)$ & $3.841 * *$ \\
\hline OR (IC 95\%) & & $2,14(1,32-3,50)$ & $1,94(1,22-3,11)$ & $2,90(1,08-10,94)$ & \\
\hline \multicolumn{6}{|l|}{$p<0,01$} \\
\hline Paridade $=1$ & $1.244(60,2)$ & $640(80,6)$ & $664(84,5)$ & $162(83,5)$ & \\
\hline Paridade $\geq 2 *$ & $823(39,8)$ & $154(19,4)$ & $122(15,5)$ & $32(16,5)$ & $3.841 * *$ \\
\hline OR (IC 95\%) & & $2,75(2,25-3,36)$ & $3,60(2,90-4,48)$ & $3,35(2,24-5,04)$ & \\
\hline \multicolumn{6}{|l|}{$p=0,000 \ldots$} \\
\hline Aborto prévio ausente & $1.844(89,2)$ & $727(91,3)$ & $727(92,5)$ & $172(88,7)$ & \\
\hline Aborto prévio presente* & $223(10,8)$ & $69(8,7)$ & $59(7,5)$ & $22(11,3)$ & 3.843 \\
\hline OR (IC 95\%) & & $1,23(0,95-1,71)$ & $1,49(1,09-2,03)$ & $0,95(0,58-1,55)$ & \\
\hline \multicolumn{6}{|l|}{$p=0,054$} \\
\hline Gestação a termo & $1.970(95,4)$ & $758(95,2)$ & $743(94,8)$ & $190(97,9)$ & \\
\hline Gestação pré-termo* & $94(4,6)$ & $38(4,8)$ & $41(5,2)$ & $4(2,1)$ & $3.838 * *$ \\
\hline OR (IC 95\%) & & $0,95(0,64-1,43)$ & $0,86(0,58-1,28)$ & $0,44(0,14-1,25)$ & \\
\hline$p=0,31$ & & & & & \\
\hline
\end{tabular}

* categoria de referência

PGI = Primeiro grau incompleto; PG C = Primeiro grau completo; $\mathrm{SG}$ = Segundo grau;

$\mathrm{TG}=$ Terceiro grau; $\mathrm{IC}=$ Intervalo de confiança; $\mathrm{OR}=$ odds ratio

** Excluídas as DN V cujos campos não estavam preenchidos para esta variável. 
desinformação pode ser responsável pela menor importância dada por estas mães aos cuidados no pré-natal e do acompanhamento médico periódico de seu filho, aumentando, assim, a possibilidade de elas, com menor escolaridade, serem multíparas.

Ao contrário de Astolfi \& Zonta (1999), este estudo não mostrou associação entre escolaridade e prematuridade, o que pode ser decorrente do fato de que as informações sobre a duração da gestação são possi velmente fornecidas erroneamente ao responsável que preenche a DNV. A informação fornecida pelo marido ou companheiro ou pelos familiares da puérpera, podem não ser fiéis ou ser ignoradas; este campo da DNV geralmente apresenta da-

\section{Referências}

ALMEIDA, R. M.; MARINS, V. M. \& VALLE, J., 1999. Breatfeeding, socio-economic conditions and nutritional status of children younger than 12 months in Brazil. Annals of Tropical Paediatrics, 19:257-262.

AL-NAHEDH, N. N., 1999. The effect of sociodemographic variables on child-spacing in rural Saudi Arabia. East Mediterranean Health Journal, 5:136140

ASTOLFI, P. \& ZONTA, L. A., 1999. Risks of preterm delivery and association with maternal age, birth order, and fetal gender. Human Reproduction, 14:2891-2894.

BOHLAND, A. K. \& JORGE, M. H. P. M., 1999. Mortalidade infantil de menores de um ano na região Sudoeste do Estado de São Paulo. Revista deSaúde Pública, 33:366-373.

CONDE-AGUDELO, A.; BELIZAN, J. M. \& DIAZROSSELLO, J. L., 2000. Epidemiology of fetal death in Latin America. Acta Obstetrica and Gynecologica Scandinavian, 79:371-378.

DEAN, A. G.; DEAN, J. A.; COULOMBIER, D.; BRENDEL, K. A.; SMITH, D. C.; BURTON, A. H.; DICKER, R. C.; SULLIVAN, K.; FAGAN, R. F. \& ARNER, T. G., 1994. Epi Info, Version 6.01: A Word Processing, Database and Statistics Program for Epidemiology on Microcomputers. Atlanta: Centers for Disease Control and Prevention.

GOULD, J. B.; DAVEY, B. \& STAFFORD, R. S., 1989. Socioeconomic differences in rates of cesarean section. New England Journal of Medicine, 321:233239.

LEKEA-KARANIKA, V.; TZOUMAKA-BAKOULA, C. \& MATSANIOTIS, N. S., 1999. Sociodemographic determinants of low birthweight in Greece: A population study. Paediatric and Perinatal Epidemiology, 13:65-77. dos conflitantes quando se compara com o peso ao nascer, por exemplo.

A associação entre aborto e escolaridade ficou, na análise de tendência, no limiar da significância estatística; essa informação também costuma ser de difícil obtenção pelo próprio tema e, para se tirar esse possível vício, a solução seria entrevistar diretamente a puérpera.

Concluindo, pode-se afirmar que a baixa escolaridade materna é um fator importante que pode predispor ao aparecimento de situações potencialmente de risco para a mãe e o recém-nascido, pois está associada ao baixo peso ao nascer, à perimortalidade, neomortalidade e mortalidade infantil, assim como ao aumento do número de partos.
NAJDAWI, F. \& FAOURI, M ., 1999. Maternal smoking and breastfeeding. East Mediterranean Health Journal, 5:450-456.

NASCIMENTO, L. F. C., 1997. Grau de instrução materna: Um marcador obstétrico. In: V Congresso Brasileiro de Saúde Coletiva, Resumos, p. 271. Águas de Lindóia: ABRASCO.

OKOLO, S. N.; ADEWUNMI, Y. B. \& OKONJI, M. C., 1999. Current breatfeeding knowledge, attitude, and practices of mothers infive rural communities inthe Savanah region of Nigeria. Journal of Tropical Pediatrics, 45:323-326.

OKOSUN, I. S.; HALBACH, S. M.; DENT, M. M. \& COOPER, R. S., 2000. Ethnic differences in teh rates of low birth weight attributable to differences in early motherhood: A study from the Third National Health and Nutrition Examination Survey. Journal of Perinatology, 20:105-109.

ORACH, C. G., 2000. Maternal mortality estimated using Sisterhood method in gulu district, Uganda. Tropical Doctor, 30:72-74.

OSBORN, J. F.; CATTARUZZA, M. S. \& SPINELLI, A., 2000. Risk of spontaneous abortion in Italy, 19781995, and effect of maternal age, gravidity, marital status, and education. American Journal of Epidemiology, 151:98-105.

SES-SP (Secretaria de Estado da Saúde de São Paulo), 1998. Mortalidade Infantil segundo as Coordenadorias e Regiões de Saú de, Estado de São Paulo, 1998. 22 de junho de 2000 \ttp:// www.saude.sp. gov.br/DSAUDE/MORB_MORB/Minf/HTML/mi creg.htm>.

THEME FI LHA, M. M.; SILVA, R. \& NORONHA, C. P., 1999. Mortalidade materna no Município do Rio de Janeiro, 1993 a 1996. Cadernos deSaúde Pública, 15:397-403. 